\title{
ON THE FINITENESS OF FREE (UNIVERSAL) ALGEBRAS
}

ALFRED L. FOSTER

1. Introduction. In the present note we exhibit a generalizationto universal algebras - of the familiar structure of free Boolean algebras, particularly the free algebras of a finite number of generators. The result here established is a ready consequence of the principal structure theorem for (universal) "ß-algebras" (Foster $[1 ; 2]$; see also below); from these references we recall several orienting essentials.

Let $S p=\left(n_{1}, n_{2}, \cdots\right)$ be a given species and $\mathfrak{U}=\left(A, o_{1}, o_{2}, \cdots\right)$ a (universal) algebra of species $S p$; here $A=\{\cdots, \xi, \cdots\}$ is the class of elements of $\mathscr{U}$ and $o_{1}, o_{2}, \cdots$ the primitive operations, where $o_{i}$ is $n_{i}$-ary,

$$
o_{i}\left(\xi_{1}, \cdots, \xi_{n_{i}}\right) \in A, \quad\left(\xi_{1}, \cdots, \xi_{n_{i}} \in A ; i=1,2, \cdots\right) .
$$

$\mathfrak{Q}$ is called "functionally strictly complete" (f.s.c.) if for each positive integer $k$ and for each mapping $f\left(\xi_{1}, \cdots, \xi_{k}\right)$ of the set $A^{(k)}(=k$ th Cartesian power of $A$ ) into the set $A$, the function $f\left(\xi_{1}, \cdots, \xi_{k}\right)$ is expressible as a "strict $\mathfrak{A}$-function," i.e., as some composition, via the primitive $o_{i}$ of $\mathfrak{A}$, of indeterminates $\xi_{1}, \cdots, \xi_{k}$ over the set $A$. If $\mathfrak{A}$ is f.s.c. it is necessarily of finite order $n$ ( $A=$ class of $n$ elements); also for at least one $n_{i}$ of $S p, n_{i}>1$ (see $[1 ; 2]$ ).

A "primal" (universal) algebra $\mathfrak{B}=\left(P, o_{1}, o_{2}, \cdots\right)$-of species $S p$ -is one which is f.s.c. and not isomorphic with $1^{\circ}$, the one-element algebra. Among the profusion of known primal algebras we here recall $\left(1^{\circ}\right)\left(F_{2}, \times, \frown\right)$, the two element Boolean algebra, and more generally $\left(2^{\circ}\right)\left(F_{p}, X, \frown\right)$, where $\times, \frown$ are respectively the product and $\xi=1+\xi$ in $\left(F_{p}, \times,+\right)$, the prime field of characteristic $p$. Again, for any integer $n>1,\left(3^{\circ}\right)\left(P_{n}, \times, \frown\right)$, the basic Post algebra of order $n$ (Rosenbloom [4]; Foster $[1 ; 2]$ ). Further we have $\left(4^{\circ}\right)$, $\left(G_{n}, \times, \frown\right)$, any "group frame" : here $\left(G_{n}, X\right)$ is $\left(G^{\prime}, X\right)$-any finite group of order $n-1$ and with its identity denoted by 1 -augmented by a null element, 0 , where $0 \times \xi=\xi \times 0=0(\xi \in G)$, and $\xi$ is defined as the cyclic permutation of $G$ corresponding to any given ordering $0,1, \alpha, \beta, \cdots, \gamma$ of $G$, i.e., $0^{-}=1,1-\alpha, \alpha=\beta, \cdots, \gamma=0$. (For these and additional primal algebras see $[1 ; 2]$.)

Each primal algebra $\mathfrak{B}$ is the "kernel" of the class of " $\mathfrak{B}$-algebras," that is, the class of algebras $\mathfrak{A}$, of the same species as $\mathfrak{B}$, which

Received by the editors November 12, 1955. 
satisfy all identities satisfied by $\mathfrak{B}$. In particular the class of all (i) Boolean algebras, (ii) $p$-algebras (rings), (iii) Post algebras, is coextensive, up to isomorphisms, with the class of all $\mathfrak{B}$-algebras with the kernel $\mathfrak{P}$ respectively chosen as (i) $\left(F_{2}, \times, \frown\right)$, (ii) $\left(F_{p}, \times, \frown\right)$, (iii) $\left(P_{n}, \times, \frown\right)$.

From [2] we require the following basic result.

Theorem 1. Let $\mathfrak{B}$ be any primal algebra. Then: (1) The class of all $\mathfrak{B}$-algebras is coextensive with the class of all subdirect powers of $\mathfrak{B}$, or, equivalently, with the class of all subalgebras of direct powers of $\mathfrak{B}$. (2) $A$ finite $\mathfrak{P}$-algebra, $\mathfrak{A}$, is isomorphic with a direct power of $\mathfrak{B}$,

$$
\mathfrak{A} \cong \mathfrak{B}^{(t)}=\mathfrak{B} \times \mathfrak{B} \times \cdots(=\text { th } \text { direct power }),
$$

and

$$
n^{\prime}=n^{\ell} \quad\left(n^{\prime}, n=\text { orders of } \mathfrak{A}, \mathfrak{B}\right) .
$$

2. Free algebras. Let $\subseteq$ be a class of equations, of species $S p$. A model of $\mathfrak{S}$, i.e., an algebra $\mathfrak{A}$ of species $S p$, which satisfies all identities $\mathfrak{S}$, is called "exact" if each identity satisfied by $\mathfrak{A}$ is a logical consequence of $\mathfrak{S}$.

Theorem 2. Let $\Im$ be a class of equations of species $S p$, let $k$ be an integer and let $\mathfrak{F}_{k}(\mathfrak{S})$ be the free algebra of $k$ generators determined by $\mathfrak{S}$. If $\subseteq$ possesses a (finite) model, $\mathfrak{B}$, which is $\left(1^{\circ}\right)$ exact and $\left(2^{\circ}\right)$ functionally strictly complete, then $\mathfrak{F}_{k}(\mathfrak{S})$ is a finite algebra with the structure

$$
\begin{aligned}
\mathfrak{F}_{k}(\mathfrak{S}) \cong \mathfrak{B}^{\left(n^{k}\right)} \quad\left(=n^{k} \text { th direct power of } \mathfrak{B} ; n=\text { order of } \mathfrak{B}\right), \\
n^{\prime}=n^{{ }^{k}} \quad\left(n^{\prime}=\text { order of } \mathfrak{F}_{k}(\mathfrak{S})\right) .
\end{aligned}
$$

Proof. Let $\xi_{1}, \cdots, \xi_{k}$ be generators of $\mathfrak{F}_{k}(\Im)$. Since $\mathfrak{B}$ is assumed to be an exact model of $\mathfrak{S}$, the elements of $\mathfrak{F}_{k}(\mathfrak{S})$ are in 1-1 correspondence with the classes $C_{1}, C_{2}, \cdots$ of identical strict $\mathfrak{B}$-functions $\mathfrak{f}\left(\xi_{1}, \cdots, \xi_{k}\right)$, i.e., $\mathfrak{f}\left(\xi_{1}, \cdots, \xi_{k}\right)$ and $\mathfrak{g}\left(\xi_{1}, \cdots, \xi_{k}\right)$ belong to the same $C_{i}$ if and only if $\mathfrak{f}=g$ is an identity of $\mathfrak{B}$. Now since $\mathfrak{P}=(P, o, \cdots)$ is further assumed to be functionally strictly complete, each of the $n^{n^{k}}$ different set-theoretical mappings $f\left(\xi_{1}, \cdots, \xi_{k}\right)$ of the set $P^{(k)}$ ( $=k$ th Cartesian power of $P$ ) into the set $P$ may be expressed in at least one way as a strict $\mathfrak{B}$-function $f\left(\xi_{1}, \cdots, \xi_{k}\right)$. Hence there are precisely $n^{n^{k}}$ classes $C_{i}$ and consequently

$$
n^{\prime}=\text { order of } \mathcal{F}_{k}(\Im)=n^{n^{k}} \text {. }
$$

In particular $\mathcal{F}_{k}(\Im)$ is thus a finite $\mathfrak{B}$-algebra. From (2) of Theorem 1 we then have: for some integer $t$,

$$
\mathfrak{F}_{k}(\Im) \cong \mathfrak{P}^{(t)}(=t \text { th direct power of } \mathfrak{B})
$$


Comparing the order of $\digamma_{k}(\Im)$ computed from (2) with (1) we have $t=n^{k}$ and the theorem is proved.

REMARK. The finiteness of $\mathcal{F}_{k}(\Im)$-apart from its structure-follows from condition $\left(1^{\circ}\right)$ alone. In fact if $\mathfrak{S}$ possesses a finite and exact model, $\mathfrak{A}$, of order $n$, the number of classes $C_{i}$ above is $\leqq n^{n^{k}}$, and we have

$$
n^{\prime}=\text { order of } \mathcal{F}_{k}(\Im) \leqq n^{n} \text {. }
$$

If in Theorem 2 we take $\subseteq$ as the class $\mathscr{g}(\mathfrak{B})$ of all strict identities satisfied by $\mathfrak{P}$ or, what is equivalent, as any basis $I_{1}, I_{2}, \cdots$ for the class $\mathscr{g}(\mathfrak{B})$, Theorem 2 may be restated in the form of Theorem 3 below. (In this connection it is known (Yaqub [5]) that $\mathfrak{g}(\mathfrak{B})$ always possesses a finite basis, i.e., a finite number of strict identities $I_{1}$, $I_{2}, \cdots, I_{s}$ satisfied by $\mathfrak{B}$ from which all strict identities satisfied by $\mathfrak{B}$ follow as a logical consequence.)

THEOREM 3. Let $\mathfrak{P}=(P, o, \cdots)$ be an arbitrary primal algebra of order $n$. Then $\mathfrak{F}_{k}[\mathfrak{B}]$, the free $\mathfrak{B}$-algebra of a finite number $k$ of generators, has the structure

$$
\begin{aligned}
\mathfrak{F}_{k}[\mathfrak{P}] & \cong \mathfrak{P}^{\left(n^{k}\right)}\left(=n^{k} \text { th direct power of } \mathfrak{P}\right), \\
n^{\prime} & =\text { order of } \mathcal{F}_{k}[\mathfrak{P}]=n^{n} .
\end{aligned}
$$

On proper choice of the kernel, $\mathfrak{B}$, Theorem 3 applies, in particular , to free Boolean algebras, free $p$-algebras (rings), free Post-algebras, etc. $(\$ 1)$.

Finally we remark that Theorem 3 (or 2) may be further generalized to yield a corresponding structure for $\mathfrak{F}_{k}\left[\mathfrak{B}_{1}, \mathfrak{B}_{2}, \cdots, \mathfrak{B}_{8}\right]$, the free " $\left(\mathfrak{B}_{1}, \cdots, \mathfrak{B}_{s}\right)$-algebra." This extension requires the general structure theorem for such algebras, derived in [3]. Here $\mathfrak{B}_{1}, \mathfrak{P}_{2}, \cdots$,

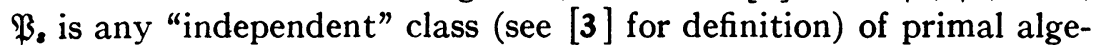

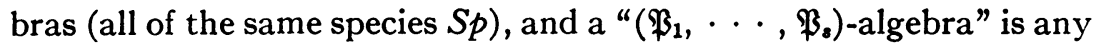
algebra, $\mathfrak{A}$, (of species $S p$ ) which satisfies all strict identities common to $\mathfrak{B}_{1}, \cdots, \mathfrak{B}_{8}$. We shall however not enter into this here.

\section{BIBLIOGRAPHY}

1. A. L. Foster, Generalized "Boolean" theory of universal algebras, Part I: Sub" direct sums and normal representation theorem, Math. Zeit. vol. 58 (1953) pp. 306-336.

2. - Generalised "Boolean" theory etc., Part II: Identities and subdirect sums of functionally complete algebras, Math. Zeit. vol. 59 (1953) pp. 191-199.

3. - The identities of-and unique subdirect factorization within-classes of universal algebras, Math. Zeit. vol. 62 (1955) pp. 171-188.

4. P. Rosenbloom, The elements of mathematical logic, The Dover Series, 1950.

5. A. Yaqub, On the theory of ring-logics and universal algebras, Doctoral dissertation, University of California, Berkeley, 1955.

University of California, Berkeley 